\title{
Spin fluctuation and the transport mechanism in vanadium oxide spinels with a metal-insulator transition
}

\author{
Masashige Onoda, Hiroyoshi Imai, Yasushi Amako, ${ }^{*}$ and Hiroshi Nagasawa \\ Institute of Physics, University of Tsukuba, Tennodai, Tsukuba 305, Japan
}

(Received 27 February 1996; revised manuscript received 2 December 1996)

\begin{abstract}
Spin fluctuation and the transport mechanism in the spinel systems $\mathrm{Li}_{x} \mathrm{Mg}_{1-x} \mathrm{~V}_{2} \mathrm{O}_{4}$ and $\mathrm{Li}_{x} \mathrm{Zn}_{1-x} \mathrm{~V}_{2} \mathrm{O}_{4}$ with $0 \leqslant x \leqslant 1$ have been studied through measurements of $\mathrm{x}$-ray diffraction, electrical resistivity, thermoelectric power, magnetization, and nuclear magnetic resonance. These compounds range from being antiferromagnetic and insulating for $\mathrm{MgV}_{2} \mathrm{O}_{4}$ (Mott type) accompanied with a structural transition to the metallic state of $\mathrm{LiV}_{2} \mathrm{O}_{4}$ with no magnetic order. The metal-insulator transition may be of Anderson type and occurs in the vicinity of $x_{c}=0.4$. The coherence length of the wave function of hole carriers in the variable-range-hopping regime has a critical exponent -1.3 against $\left|x-x_{c}\right|$. The metallic phase above $x_{c}$ may have two kinds of carriers from dynamic mixed valence state of $\mathrm{V}^{3+}$ and $\mathrm{V}^{4+}$. Based on the magnetic susceptibility and relaxation analyses, metallic compounds may be considered to be highly correlated electron systems with a low degeneracy temperature or large mass enhancement. On the other hand, insulators have short-range ordered spin correlation and/or superparamagnetic effects. At low temperatures, an antiferromagnetic phase is realized for $x$ $\leqslant 0.05$ and a spin-glass phase originating from the frustration inherent in the spinel $B$ lattice appears in the region of $0.07 \leqslant x \leqslant 0.7$. The latter phase is enhanced for concentrations slightly less than $x_{c}$. [S0163-1829(97)02931-7]
\end{abstract}

\section{INTRODUCTION}

Many vanadium oxides and bronzes have been studied in order to investigate peculiar behaviors in correlated-electron or electron-phonon-coupling systems. Especially, a mechanism for the metal-insulator transition, hereafter referred to as MIT, induced by changing the temperature or the electron density has been explored for a long time. ${ }^{1,2}$ It is not easy to clarify the driving force for this transition, because the MIT has often been accompanied by a change in the crystal symmetry. The so-called Mott transition is essentially a bandcrossing transition and may be discontinuous in $U / W, U$ and $W$ being the effective electron-electron correlation energy and the bandwidth, respectively. ${ }^{1}$

Vanadium oxides with a chemical formula $M \mathrm{~V}_{2} \mathrm{O}_{4}$, where $M$ is $\mathrm{Li}, \mathrm{Mg}$, or $\mathrm{Zn}$, etc., have a normal spinel structure. ${ }^{3,4}$ The unit cell is usually taken to be cubic with eight molecules of $M \mathrm{~V}_{2} \mathrm{O}_{4}$ and the space group is $F d \overline{3} m$. The $M$ and $\mathrm{V}$ ions are located at the oxygen tetrahedral $A(8 a)$ and octahedral $B(16 d)$ sites, respectively. The $\mathrm{VO}_{6}$ octahedra share edges and three arrays of the $\mathrm{V}$ ions cross at each site. Each $\mathrm{V}$ ion has six neighboring ones.

In the $\mathrm{Li}_{x} \mathrm{Zn}_{1-x} \mathrm{~V}_{2} \mathrm{O}_{4}$ system, hereafter referred to as LZVO, the $\mathrm{Li}_{x} \mathrm{Zn}_{1-x}$ ion is considered to be located at the $A$ site and an average valence of $\mathrm{V}$ ions is expressed as 3 $+x / 2$. Metallic properties of $\mathrm{LiV}_{2} \mathrm{O}_{4}$ down to low temperatures have been revealed by the electrical resistivity for the single crystal specimens, ${ }^{5}$ although the density of states at the Fermi level appears to be considerably low. ${ }^{6}$ On the other hand, $\mathrm{ZnV}_{2} \mathrm{O}_{4}$ or $\mathrm{MgV}_{2} \mathrm{O}_{4}$ with $\mathrm{V}^{3+}$ is a Mott insulator or semiconductor. ${ }^{6-8}$ The antiferromagnetic transitions appear at about $75 \mathrm{~K}$ for $\mathrm{MgV}_{2} \mathrm{O}_{4}$ and at $60 \mathrm{~K}$ for $\mathrm{ZnV}_{2} \mathrm{O}_{4}$ accompanied with a cubic-tetragonal transition and specific-heat anomaly. ${ }^{8,9}$ The ordered phase has also been analyzed. ${ }^{10}$
Here, we should note that the characteristic spinel structure with cubic symmetry leads to a high degree of topological frustration for antiferromagnetically correlated spins. A substitution of $\mathrm{V}^{3+}$ by nonmagnetic $\mathrm{Al}^{3+}$ introduces a spin-glass phase, ${ }^{8,9}$ which may be due to a partial lift of the degenerated ground state as postulated theoretically. ${ }^{11}$

The electronic states of LZVO with $x \geqslant 0.4$ is essentially metallic and the MIT is based on a variable-range-hopping (VRH) or percolation model. ${ }^{7}$ In the vicinity of the MIT point, no crystal symmetry varies. In the temperature region above $500 \mathrm{~K}$, there is no essential difference between the magnetic properties of metals and insulators, and Hund's rule holds with a common antiferromagnetic superexchange coupling constant. ${ }^{12}$ However, both the Curie constant and Weiss temperature below $500 \mathrm{~K}$ have different values from those above $500 \mathrm{~K}$ probably due to the conduction electrons.

The present authors' group has examined the percolation model of LZVO from a microscopic point of view under several assumptions. ${ }^{13}$ Two kinds of nuclear magnetic resonance (NMR) lines for the $\mathrm{V}$ ion have been observed around $x=0.4$, these being roughly similar to the lines of metallic $\mathrm{LiV}_{2} \mathrm{O}_{4}$ and insulating $\mathrm{ZnV}_{2} \mathrm{O}_{4}$. In addition, two kinds of $\mathrm{Li}$ NMR lines have been observed in the region below $x=0.1$. Thus, we have postulated that two kinds of electronic pathways coexist and the valence of $\mathrm{V}$ ions depends on the nearest-neighbor configuration of $\mathrm{Li}^{+}$or $\mathrm{Zn}^{2+}$.

In this paper, we shall discuss spin fluctuation and transport mechanism in the $\mathrm{Li}_{x} \mathrm{Mg}_{1-x} \mathrm{~V}_{2} \mathrm{O}_{4}$ system with $0 \leqslant x$ $\leqslant 1$, hereafter referred to as LMVO, through measurements of x-ray diffraction, electrical resistivity, thermoelectric power, magnetization, and NMR. The NMR relaxation results for LZVO are also presented. Since the LMVO and LZVO are isomorphous, the electronic states are expected to be similar. Previous transport and magnetization measure- 
ments of the LZVO have been done above $100 \mathrm{~K}$ at $x$ intervals of $0.1{ }^{7,12}$ This work provides detailed results that include data at lower temperatures as well as for the compounds with $0<x<0.1$ near the Mott insulator. The experimental methods are given in Sec. II. Section III describes the experimental results and summarizes basic items with respect to the structure and electronic states of vanadium spinels, referring properly to previous analysis procedures. In Sec. IV, the structural, transport, and magnetic properties clarified by this work are discussed and the phase diagram at low temperatures presented. Finally, Sec. V is devoted to a conclusion for spin fluctuation and transport mechanism in LMVO and LZVO.

\section{EXPERIMENTS}

Sintered specimens of the $\mathrm{Li}_{x} \mathrm{Mg}_{1-x} \mathrm{~V}_{2} \mathrm{O}_{4}$ system were prepared by the solid state reaction method. First, $\mathrm{Li}_{3} \mathrm{VO}_{4}$ was prepared by heating a mixture of $3 \mathrm{Li}_{2} \mathrm{CO}_{3}(99.99 \%$ purity) and $\mathrm{V}_{2} \mathrm{O}_{5}(99.99 \%$ purity) in air at $953 \mathrm{~K}$ for $48 \mathrm{~h}$ and $\mathrm{V}_{2} \mathrm{O}_{3}$ was reduced by heating $\mathrm{V}_{2} \mathrm{O}_{5}$ in an $\mathrm{N}_{2} / \mathrm{H}_{2}$ atmosphere at $973 \mathrm{~K}$ for $12 \mathrm{~h}$. Appropriate mixtures of these compounds and $\mathrm{MgO}$ (99.99\% purity) were ground and pressed into pellets. They were inserted in alumina crucibles and sealed in quartz tubes, which were heated at $953-993 \mathrm{~K}$ for $48-72 \mathrm{~h}$. An oxygen deficient specimen $\mathrm{LiV}_{2} \mathrm{O}_{3.92}$ was also prepared. The specimens of $\mathrm{Li}_{x} \mathrm{Zn}_{1-x} \mathrm{~V}_{2} \mathrm{O}_{4}$ were prepared according to the same procedure as described in Ref. 7.

An x-ray powder diffraction with a $\mathrm{Cu} K \alpha$ radiation was measured on a Rigaku RAD-IIC two-circle diffractometer at about $300 \mathrm{~K}$. Electrical resistivity was taken by a dc fourprobe method in the region between 4.2 and $300 \mathrm{~K}$. The electrodes were made by using a silver paint. Thermoelectric power was taken by a dc method in the region between 4.2 and $350 \mathrm{~K}$. Gold wire of $0.02 \mathrm{~mm} \phi$ was used as potential leads and contacted to the specimens with a gold paint. Temperature gradients less than $1 \mathrm{~K}$ were applied.

Magnetization was measured by the Faraday method in the region between 4.2 and $900 \mathrm{~K}$, where fields of up to about $10 \mathrm{kG}$ were applied. The susceptibility was estimated from the linear coefficient of magnetization versus field $(M-H)$ curve in the decreasing process of the field. The magnetization was also measured under field-cooled (10 kG) and zero-field-cooled conditions, hereafter referred as to FC and ZFC, respectively.

A continuous-wave NMR measurement for the ${ }^{51} \mathrm{~V}$ and ${ }^{7} \mathrm{Li}$ nuclei in $\mathrm{LiV}_{2} \mathrm{O}_{4}$ was carried out in the region between 10 and $570 \mathrm{~K}$ on a Varian spectrometer. Frequencies from 3 to $32 \mathrm{MHz}$ and those from 6 to $22 \mathrm{MHz}$ were used to measure the field dependence of the Li and V NMR signals, respectively. The external field was calibrated by measuring the $\mathrm{Na}$ and $\mathrm{V}$ resonance signals in aqueous $\mathrm{NaVO}_{3}$ solution and the $\mathrm{Li}$ one in aqueous $\mathrm{LiCl}$ solution, where the $\mathrm{V}$ and $\mathrm{Li}$ signals were used as the reference positions of zero resonance shift.

An NMR relaxation measurement was done in the region between 77 and $430 \mathrm{~K}$ with the use of a Bruker SXP spectrometer. Signals of both $\mathrm{V}$ and $\mathrm{Li}$ were taken for $\mathrm{LiV}_{2} \mathrm{O}_{4}$, while for other compounds of the LZVO, only the Li signal was measured. The frequencies of $13.5,13.8$, and $19.8 \mathrm{MHz}$

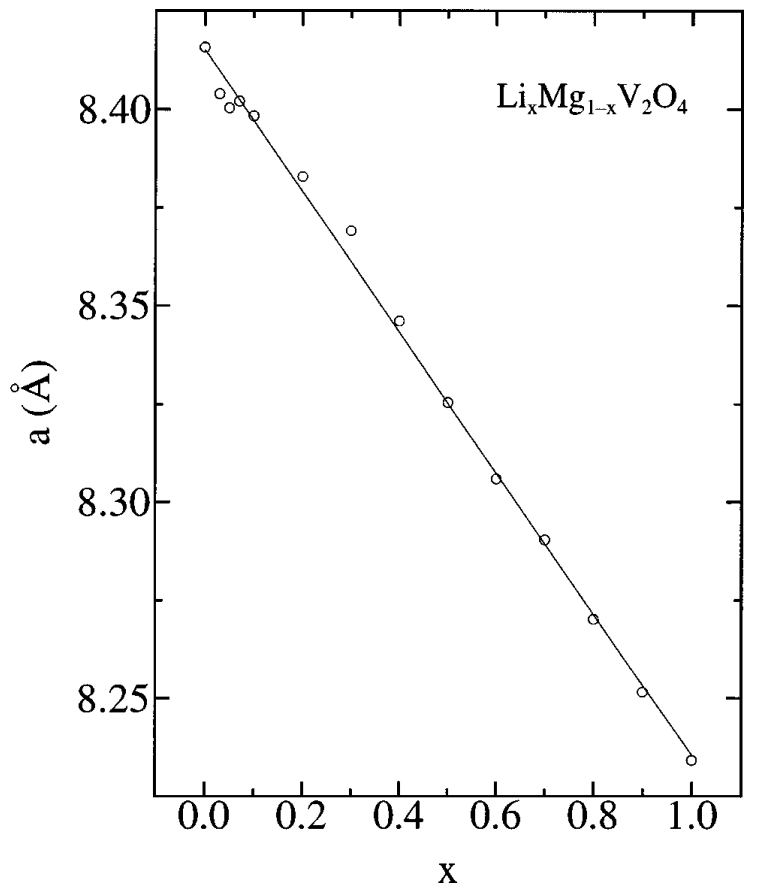

FIG. 1. $x$ dependence of the lattice constant $a$ of $\mathrm{Li}_{x} \mathrm{Mg}_{1-x} \mathrm{~V}_{2} \mathrm{O}_{4}$, where the solid line indicates the least-squares fit, $a=8.4151-0.1797 x$.

were used. The spin-lattice relaxation times were determined by the saturation comb-pulse method.

\section{EXPERIMENTAL RESULTS}

\section{A. X-ray diffraction}

X-ray powder diffraction patterns reveal a normal spineltype single phase for all $x$. The cubic lattice constant $a$ is shown in Fig. 1 in relation to $x$ and the intensity ratios for several reflections are in Fig. 2. The lattice constant is nearly proportional to $x$. Therefore, the random occupation in a macroscopic scale at the $A$ site by the $\mathrm{Li}$ and $\mathrm{Mg}$ ions may be expected from a Vegard's law as in the case of LZVO. ${ }^{7}$ This may be possible, because the effective ionic radii of $\mathrm{Li}^{+}$, $\mathrm{Mg}^{2+}$, and $\mathrm{Zn}^{2+}$ with an oxygen tetrahedral coordination are $0.59,0.57$, and $0.60 \AA$, respectively. ${ }^{14}$

\section{B. Transport phenomena}

At the bottom of Fig. 3, the electrical resistivity $\rho$ is plotted based on a single activation energy model, $\rho$ $=\rho_{0} \exp \left(E_{g} / k T\right)$, where $\rho_{0}$ is assumed to be a constant, $E_{g}$ is the half gap between the valence- and conduction-band edges, and $k$ is Boltzmann's constant. The top indicates the $\ln \rho$ versus $T^{-1 / 4}$ plot that is a VRH model in three dimensions, $\rho=\rho_{0}^{\prime} \exp \left(T_{0} / T\right)^{1 / 4} \cdot{ }^{15,16}$ All the compounds of LMVO exhibit a semiconductorlike conduction. For only $\mathrm{MgV}_{2} \mathrm{O}_{4}$, the temperature dependence of $\rho$ is approximately expressed by the single activation energy model. From the solid line at the bottom of Fig. 3 we obtain $\rho_{0}=0.11 \Omega \mathrm{cm}$ and $E_{g} / k$ $=1.6 \times 10^{3} \mathrm{~K}$. The data between $0<x \leqslant 0.5$ are fitted in the wide temperature region in terms of the VRH model. The solid lines in the top of Fig. 3 lead to the following results for $\rho_{0}^{\prime}$ and $T_{0} ; 8.6 \times 10^{-11} \Omega \mathrm{cm}$ and $1.0 \times 10^{8} \mathrm{~K}$ for $x$ 


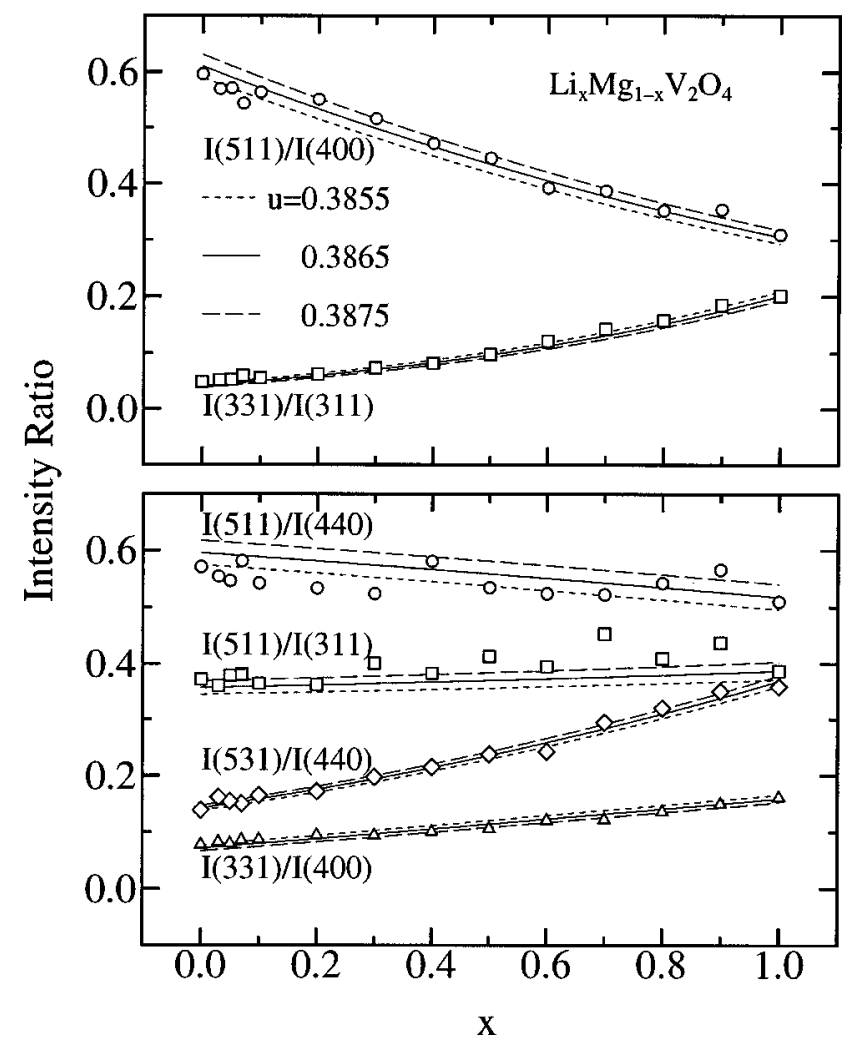

FIG. 2. $x$ dependence of the intensity ratio for several reflections of $\mathrm{Li}_{x} \mathrm{Mg}_{1-x} \mathrm{~V}_{2} \mathrm{O}_{4}$, where the dotted, solid, and dashed lines indicate the calculated results for $u=0.3855,0.3865$, and 0.3875 , respectively.

$=0.2,9.1 \times 10^{-10} \Omega \mathrm{cm}$ and $5.5 \times 10^{7} \mathrm{~K}$ for $x=0.3,9.9$ $\times 10^{-10} \Omega \mathrm{cm}$ and $4.2 \times 10^{7} \mathrm{~K}$ for $x=0.4$, and 3.5 $\times 10^{-8} \Omega \mathrm{cm}$ and $1.7 \times 10^{7} \mathrm{~K}$ for $x=0.5$. With increasing $x, T_{0}$ decreases, suggesting the wave function of the carriers extends as $x$ increases. In $x=0.6$ and 0.7 , the VRH model appears to hold only above $160 \mathrm{~K}$. The $\rho_{0}^{\prime}$ and $T_{0}$ values are estimated as $2.7 \times 10^{-7} \Omega \mathrm{cm}$ and $1.2 \times 10^{7} \mathrm{~K}$ for $x=0.6$ and $1.2 \times 10^{-5} \Omega \mathrm{cm}$ and $1.7 \times 10^{6} \mathrm{~K}$ for $x=0.7$. Neither of the previous two models can express the temperature dependence of $\rho$ for $0.8 \leqslant x \leqslant 1$. The fact that the absolute values of $\rho$ at room temperature are of the order of $10^{-1} \Omega \mathrm{cm}$ in spite of the loosely bound specimens may suggest that the compounds in this $x$ region are essentially metallic. These results are incompatible with the previous report for LZVO that states that no VRH model expresses the temperature dependence of the resistivity for $x>0{ }^{7}$

The temperature dependence of thermoelectric power $S$ is shown in Fig. 4(a). Only $S$ of $\mathrm{MgV}_{2} \mathrm{O}_{4}$ can be expressed by a wide band semiconductor model ${ }^{17}$ with hole carriers as shown by the solid line at the top of Fig. 4(a), where the gap between the valence-band edge and the Fermi energy is 520 $\mathrm{K}$ and the relaxation time of the electron momentum has an energy dependence of $\left(E-E_{v}\right)^{0.16}, E_{v}$ being the energy at the valence-band edge. If $\mathrm{MgV}_{2} \mathrm{O}_{4}$ is both an atomically and electronically homogeneous system, the energy difference between $E_{g}$ and $E_{g}^{\prime}$ may not be intrinsic and $E_{g}^{\prime} / k$ is probably more reliable. The small substitution of $\mathrm{Mg}$ by $\mathrm{Li}$ leads to a drastic change in $S$. In $0<x \leqslant 0.3, S$ depends little on temperature and its positive magnitude decreases rapidly

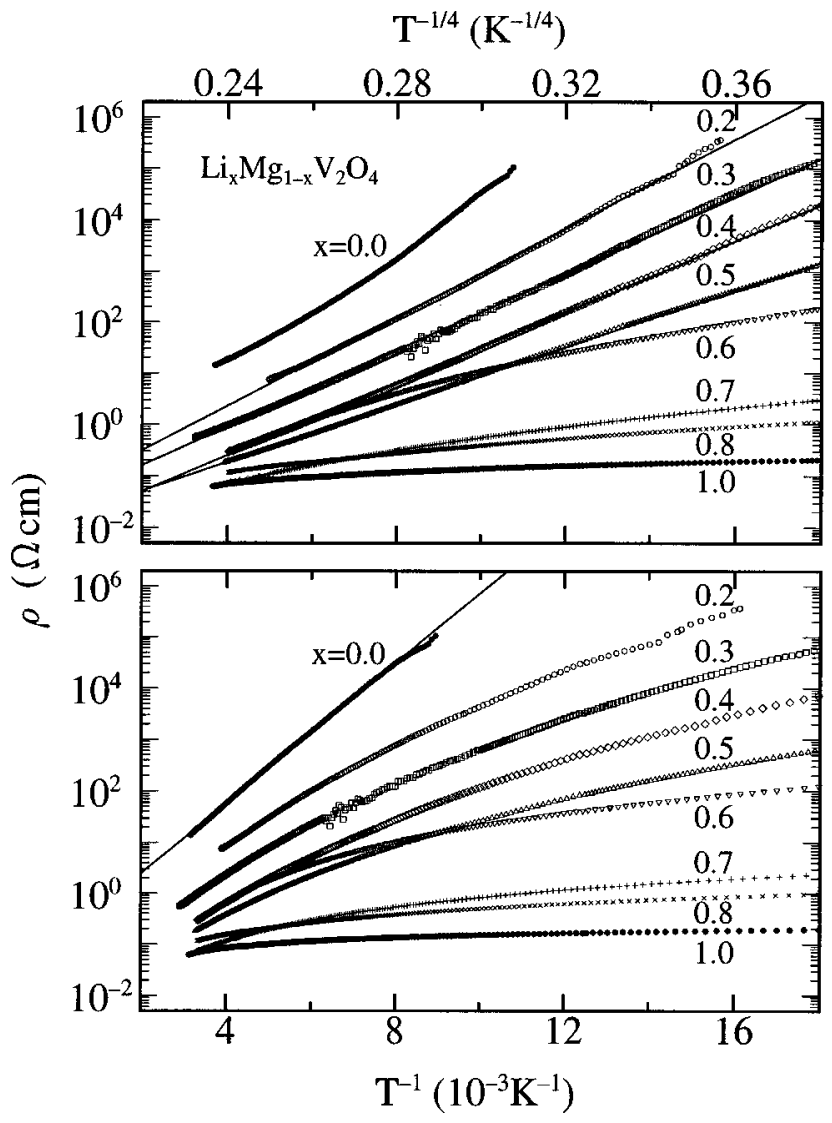

FIG. 3. Temperature dependence of the electrical resistivity $\rho$ of $\mathrm{Li}_{x} \mathrm{Mg}_{1-x} \mathrm{~V}_{2} \mathrm{O}_{4}$; the bottom is $\ln \rho$ vs $T^{-1}$ and the top is $\ln \rho$ vs $T^{-1 / 4}$ based on a variable-range-hopping model, where the solid lines indicate the calculated results with parameters provided in the text.

with $x$ as shown at the top of Fig. 4(b). This is attributed to the densely distributed energy levels around the Fermi level due to the random distribution of $\mathrm{Li}_{x} \mathrm{Mg}_{1-x}$ at the $A$ site as suggested by Kawakami, Sakai, and Tsuda. ${ }^{7}$ This appears to be similar to the idea that electrostatic forces trap holes at the $\mathrm{V}$ site nearest to $\mathrm{Li}^{+}$ion and the excited carrier from the trap will be responsible for the thermoelectric power. ${ }^{18,19}$ Judging from the behavior of $S$, we may regard the MIT point of LMVO as $x_{c}=0.4$. This corresponds to an average carrier concentration of 0.2 , which is about one-half of the critical probability of the $B$ sublattice in spinels for the connectivity of the atomic octahedral sites, in other words, the percolation threshold $p_{c}=0.401 .^{20}$ The difference between $x_{c} / 2$ and $p_{c}$ may mean that the wave function of hole carriers extends over neighboring V-V distance.

For the metallic compounds with $x \geqslant 0.4, S$ data may be classified as $0.4 \leqslant x \leqslant 0.7$ and $0.8 \leqslant x \leqslant 1$. In the former group, $S$ becomes negative at low temperatures, while in the latter, $S$ below $300 \mathrm{~K}$ is positive and remains with a small value. A characteristic peak at about $60 \mathrm{~K}$ exists in $\mathrm{LiV}_{2} \mathrm{O}_{4}$.

\section{Magnetization}

The magnetic susceptibilities $\chi$ above and below $200 \mathrm{~K}$, defined as a linear coefficient to the field, are shown in Figs. 5(a) and 5(b) as a function of temperature, respectively. A 

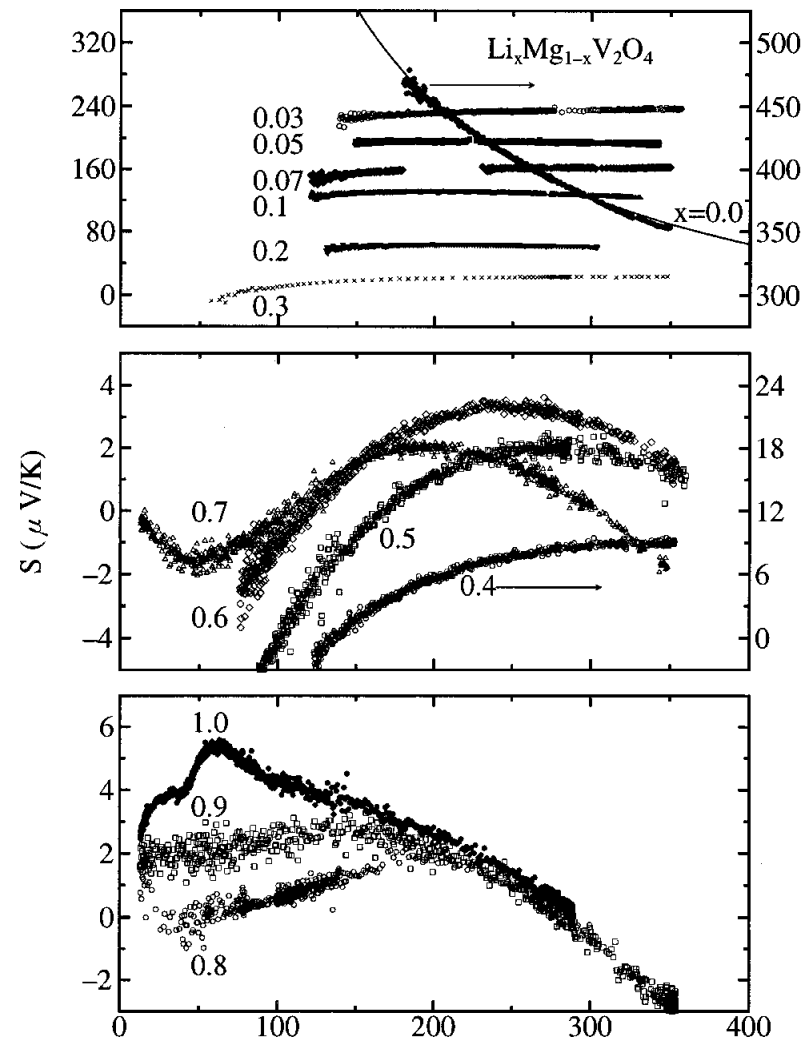

(a)

$\mathrm{T}(\mathrm{K})$
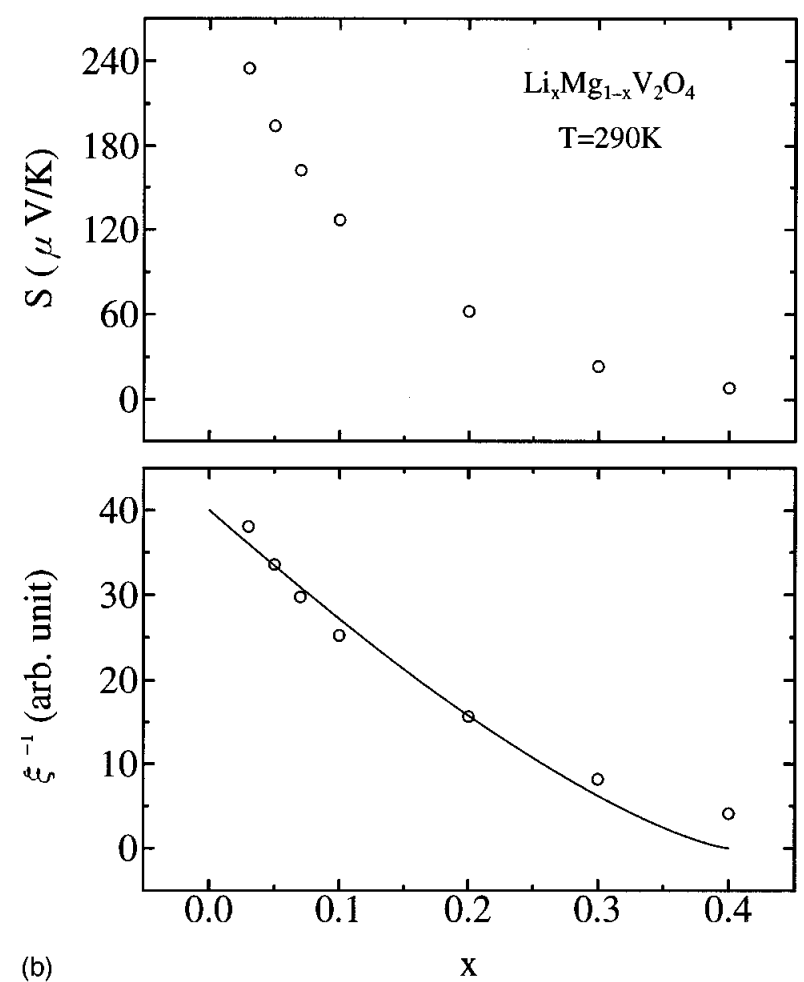

FIG. 4. (a) Temperature dependence of the thermoelectric power $S$ of $\mathrm{Li}_{x} \mathrm{Mg}_{1-x} \mathrm{~V}_{2} \mathrm{O}_{4}$, where the solid line for $\mathrm{MgV}_{2} \mathrm{O}_{4}$ indicates the calculated result based on a wide band semiconductor model with parameters given in the text. (b) $x$ dependence of the thermoelectric power $S$ at $290 \mathrm{~K}$ (top) and that of the inverse of coherence length $\xi^{-1}$ for the carriers in the variable-range-hopping regime (bottom) of $\mathrm{Li}_{x} \mathrm{Mg}_{1-x} \mathrm{~V}_{2} \mathrm{O}_{4}$, where the solid line provides the relation of $\xi^{-1} \propto|x-0.4|^{1.3}$.
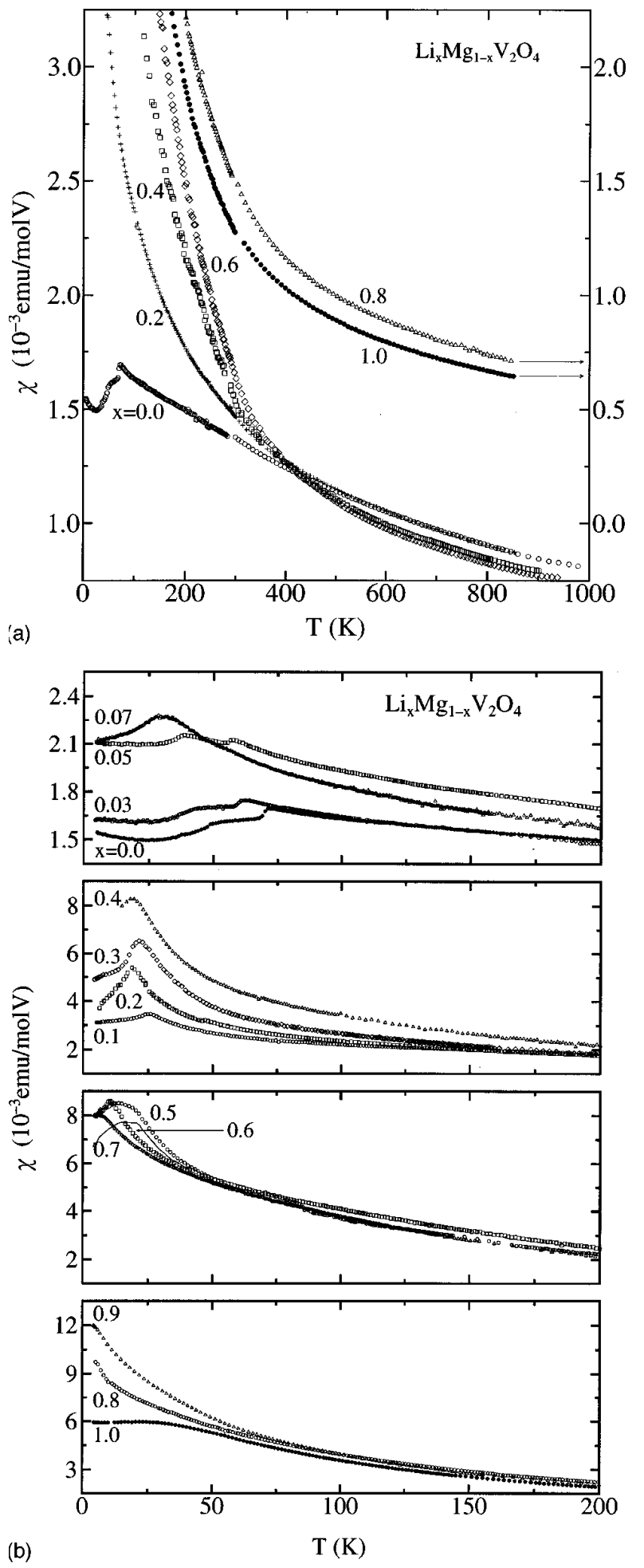

FIG. 5. (a) Temperature dependence of the magnetic susceptibility $\chi$ of $\mathrm{Li}_{x} \mathrm{Mg}_{1-x} \mathrm{~V}_{2} \mathrm{O}_{4}$ with $x=0,0.2,0.4,0.6,0.8$, and 1 above 200 K. (b) Temperature dependence of the magnetic susceptibility $\chi$ of $\mathrm{Li}_{x} \mathrm{Mg}_{1-x} \mathrm{~V}_{2} \mathrm{O}_{4}$ below $200 \mathrm{~K}$. The reversible susceptibility $\chi_{r}$ of $x=0.5$ under the field-cooled condition is expressed by the solid line.

Curie-Weiss-like temperature dependence is observed for all the compounds above $75 \mathrm{~K}$. In $\mathrm{MgV}_{2} \mathrm{O}_{4}$, there is the sharp edge at $T_{N} \simeq 75 \mathrm{~K}$ and an additional broad peak appears at $T_{g} \simeq 50 \mathrm{~K}$, which agrees with previous results for the specimens prepared in a gas flow of $\mathrm{N}_{2}$ and $\mathrm{H}_{2} .{ }^{8,9}$ Similar behav- 
iors are seen for $x=0.03$ and 0.05 , although both $T_{N}$ and $T_{g}$ are reduced largely. For $0.07 \leqslant x \leqslant 0.7$, only the broad peak is observed. In $x=0.8$ and 0.9 , there is no peak, while $\mathrm{LiV}_{2} \mathrm{O}_{4}$ has a tendency to saturate below $30 \mathrm{~K}$. This saturation behavior disappears by a small oxygen deficiency, $\mathrm{LiV}_{2} \mathrm{O}_{3.92}$.

For the FC measurements in the range $0 \leqslant x \leqslant 0.7$, there exists the remanent magnetization $\sigma$ defined as $M=\chi_{r} H$ $+\sigma, \chi_{r}$ being the reversible susceptibility. The typical temperature dependence of $\chi_{r}$ of $x=0.5$ is shown in Fig. 5(b) by the solid line. There is a clear difference between $\chi$ and $\chi_{r}$. Figure 6(a) indicates the temperature dependence of $\sigma$. The arrow in this figure denotes $T_{g}$. The temperature at which $\sigma$ appears is found to be the same as $T_{g}$. The magnitude at $6 \mathrm{~K}$ is in the range from 0.3 to $13 \mathrm{emu} / \mathrm{mol} \mathrm{V}$ as shown in Fig. 6(b). The largest value is seen around $x$ $=0.3$. However, there is no difference between the magnetization curves under FC and ZFC conditions in $0.8 \leqslant x \leqslant 1$.

The magnetic susceptibility is generally given by $\chi=$ $\chi_{d}+\chi_{\mathrm{orb}}+\chi_{\mathrm{dia}}$, where $d$, orb, and dia refer to the contributions from spin, Van Vleck orbital paramagnetism, and closed-shell ion diamagnetism, respectively, $\chi_{\text {dia }}$ being $(-35$ $+1.3 x) \times 10^{-6} \mathrm{emu} / \mathrm{mol} \mathrm{V}$ from Ref. 21 . As pointed out previously, ${ }^{12,8}$ the high-temperature data above $500 \mathrm{~K}$ indicate that $\chi_{d}$ follows the Curie-Weiss law expressed as $\chi_{d}=C /\left(T+T_{W}\right)$, where $T_{W}$ is the Weiss temperature and $C$ is the Curie constant given by $C=N g^{2} \mu_{B}^{2} S(S+1) /(3 k)$, $N, g, \mu_{B}$, and $S$ being the number of $\mathrm{V}$ ions, the $g$ factor, the Bohr magneton, and the spin number, respectively. The orbital susceptibility is estimated to be 1.0(2) $\times 10^{-4} \mathrm{emu} / \mathrm{mol} \mathrm{V}$ for $0.4 \leqslant x \leqslant 1$ and $1.8(2) \times 10^{-4} \mathrm{emu} /$ mol $\mathrm{V}$ for $0 \leqslant x \leqslant 0.3$. This difference has been also confirmed by NMR study ${ }^{22}$ and should be attributed to that of electronic structures. Figure 7 illustrates the $C \chi_{d}^{-1}$ versus $T$ plot, where $S(S+1)=2-5 x / 8$ and $g=1.98$. All the results have a straight line with an almost common slope above 500 $\mathrm{K}$. The superexchange coupling constant $J$ is approximately $x$ independent, $54 \mathrm{~K}$ from the relation $T_{W}=4 J S(S+1) / k$.

Below $500 \mathrm{~K}, C \chi_{d}^{-1}$ has a different slope from that at high temperatures. Except for the behavior below $100 \mathrm{~K}$, $\chi_{d}^{-1}$ for $0 \leqslant x \leqslant 0.3$ has an antiferromagnetic coupling constant, while that for $0.4 \leqslant x \leqslant 1$ has zero or a small ferromagnetic constant. This result for $\mathrm{LiV}_{2} \mathrm{O}_{4}$ is not consistent with those reported by other groups. ${ }^{23,24}$ It is likely caused by the difference between estimations of constant paramagnetism and/or the quality of specimens. As described above, we have determined $\chi_{\text {orb }}$ based on the precise analysis of magnetic susceptibility at high temperatures as well as the Knight shift of ${ }^{51} \mathrm{~V}$ nuclei.

\section{NMR}

\section{1. $V$ ion}

One central resonance line of the ${ }^{51} \mathrm{~V}$ nuclei with spin $\frac{7}{2}$ is observed in $\mathrm{LiV}_{2} \mathrm{O}_{4}$ as described in Ref. 13. In the frequency range from 6 to $22 \mathrm{MHz}$, no characteristic powder structures are observed. Figure 8 shows the temperature dependence of the resonance line shifts at 6 and $15 \mathrm{MHz}$. The resonance line shifts are frequency independent and thus cor-
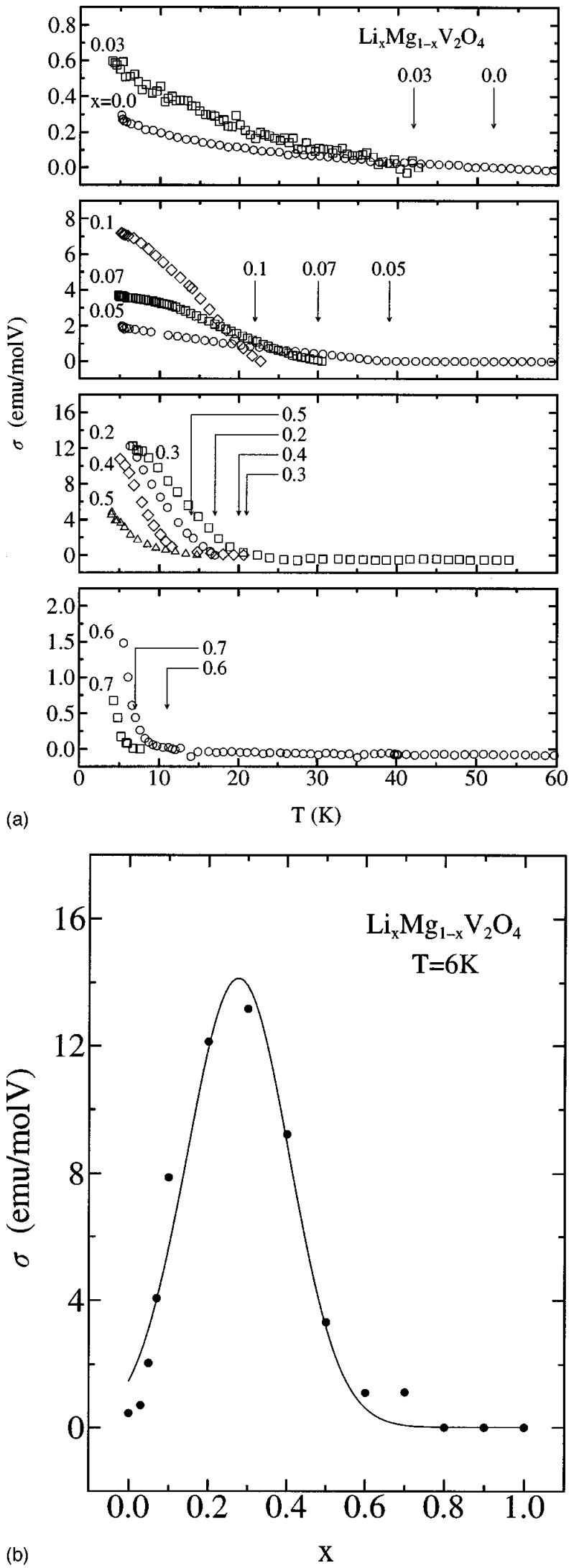

FIG. 6(a). Temperature dependence of the remanent magnetization $\sigma$ under the field-cooled condition of $\mathrm{Li}_{x} \mathrm{Mg}_{1-x} \mathrm{~V}_{2} \mathrm{O}_{4}$, where the arrow for each $x$ indicates the temperature at which the magnetic susceptibility takes a broad peak. (b) $x$ dependence of the remanent magnetization $\sigma$ at $6 \mathrm{~K}$ of $\mathrm{Li}_{x} \mathrm{Mg}_{1-x} \mathrm{~V}_{2} \mathrm{O}_{4}$, where the solid line is a guide to the eye. 


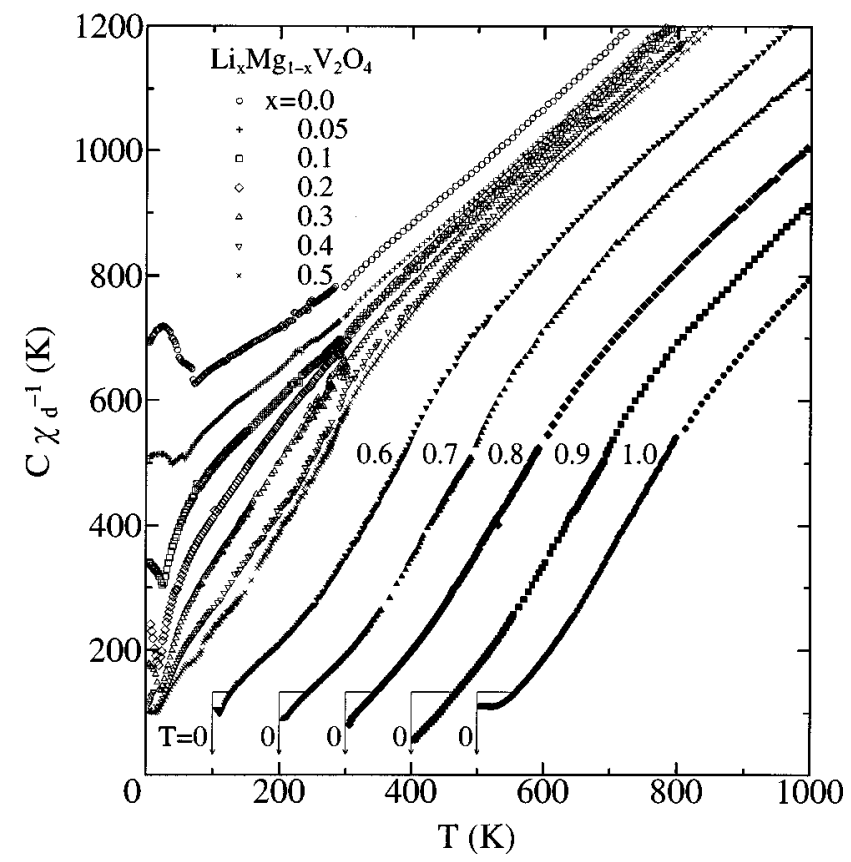

FIG. 7. Temperature dependence of the product of inverse spin susceptibility $\chi_{d}^{-1}$ and Curie constant $C$ for $\mathrm{Li}_{x} \mathrm{Mg}_{1-x} \mathrm{~V}_{2} \mathrm{O}_{4}$. For readability, the origin for the data of $x=0.6,0.7,0.8,0.9$, and 1 is shifted to the point indicated by the arrow.

respond to the Knight shifts $K$. It increases in the negative direction with decreasing temperature and reaches a minimum at about $20 \mathrm{~K}$.

The magnetization by the saturation comb pulse shows a single recovery. $\left(T_{1} T\right)^{-1}$, where $T_{1}$ is the spin-lattice relax-

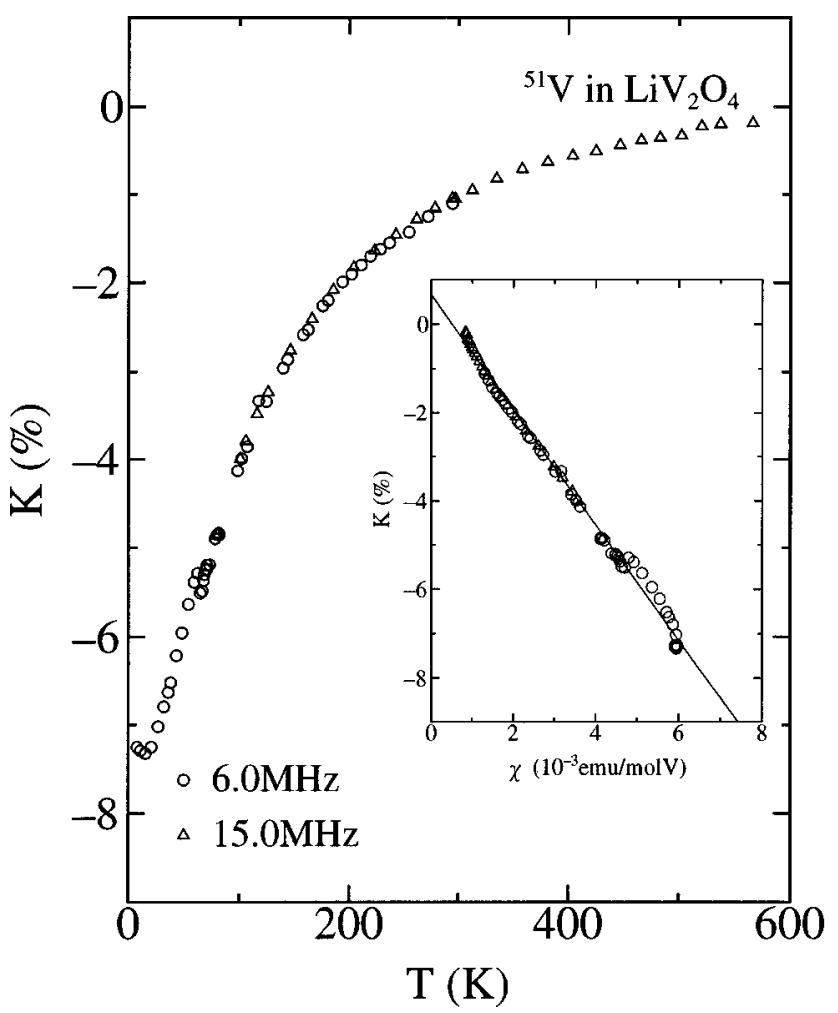

FIG. 8. Temperature dependence of the Knight shift $K$ of ${ }^{51} \mathrm{~V}$ nuclei in $\mathrm{LiV}_{2} \mathrm{O}_{4}$ at 6.0 and $15.0 \mathrm{MHz}$, where the inset shows the Knight shift vs susceptibility diagram.

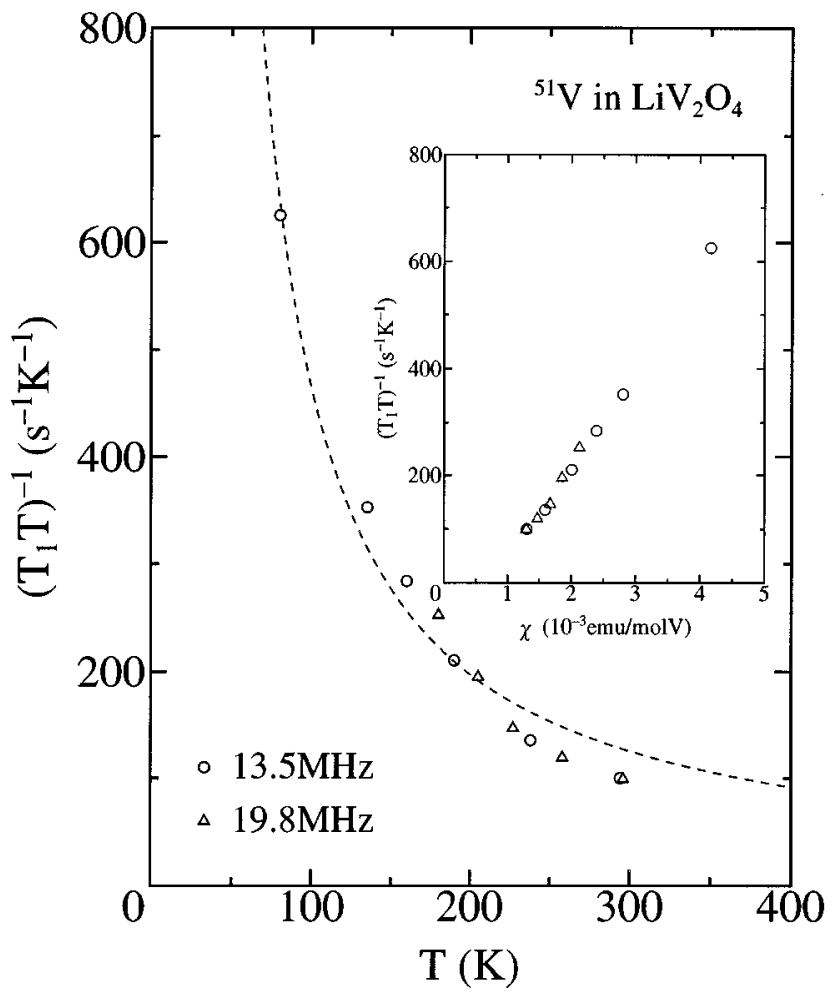

FIG. 9. Temperature dependence of the $\left(T_{1} T\right)^{-1}$ of ${ }^{51} \mathrm{~V}$ nuclei in $\mathrm{LiV}_{2} \mathrm{O}_{4}$ at 13.5 and $19.8 \mathrm{MHz}$, where $T_{1}$ is the spin-lattice relaxation time.

ation time at 13.5 and $19.8 \mathrm{MHz}$, as a function of temperature is shown in Fig. 9. It is nearly frequency independent and increases with decreasing temperature in the measured temperature region.

\section{Li ion}

One central resonance line is observed for the ${ }^{7} \mathrm{Li}$ nuclei with spin $\frac{3}{2}$ in $\mathrm{LiV}_{2} \mathrm{O}_{4}$. The resonance line shape is approximately Gaussian in the frequency range from 3 to $32 \mathrm{MHz}$. Here, modulation width less than $0.1 \mathrm{G}$ is used and its effect on the linewidths is negligible. The resonance line shifts and the full widths at half maximum $\Delta H_{\mathrm{FWHM}}$ at $6.25,15.0$, and $30.3 \mathrm{MHz}$ as a function of temperature are shown in Figs. 10 and 11, respectively. The line shift is frequency independent, so that it is thought to be the Knight shift. It increases with decreasing temperature and then reaches a maximum at about $20 \mathrm{~K}$. On the other hand, the width depends largely on the frequency. The $\Delta H_{\mathrm{FWHM}}$ at $6.25 \mathrm{MHz}$ is nearly temperature independent except for the results above $500 \mathrm{~K}$, but at the higher frequencies, it increases with decreasing temperature or increasing frequency.

The single recovery of magnetization by the saturation comb pulse in LZVO is obtained. The temperature dependence of $\left(T_{1} T\right)^{-1}$ at $19.8 \mathrm{MHz}$ is shown in Fig. 12. Except for $\mathrm{LiV}_{2} \mathrm{O}_{4}$, it increases with decreasing temperature and has a maximum around $x=0.6$. In $\mathrm{LiV}_{2} \mathrm{O}_{4}$, small anomalies are noted at approximately 140 and $270 \mathrm{~K}$.

\section{DISCUSSION}

\section{A. Structural properties}

From the intensity ratios shown in Fig. 2, the $u$ parameter of the oxygen atoms located at the $32 e$ site is estimated. ${ }^{4}$ 


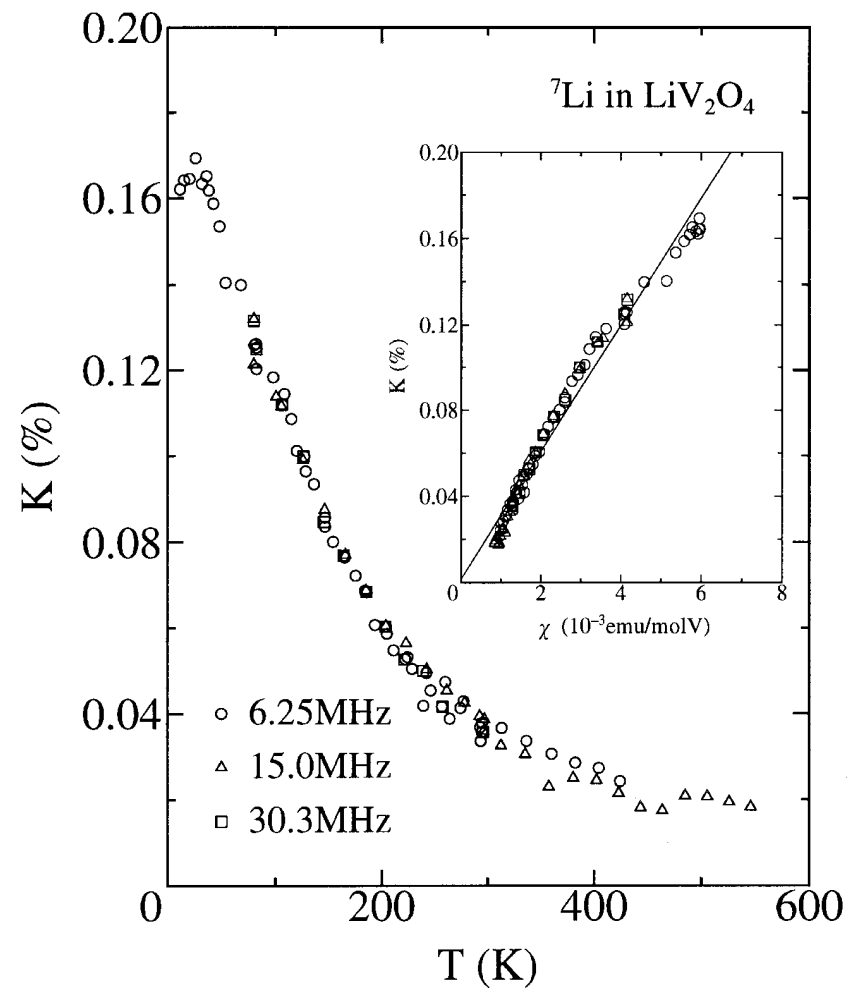

FIG. 10. Temperature dependence of the Knight shift $K$ of ${ }^{7} \mathrm{Li}$ nuclei in $\mathrm{LiV}_{2} \mathrm{O}_{4}$ at $6.25,15.0$, and $30.3 \mathrm{MHz}$, where the inset shows the Knight shift vs susceptibility diagram.

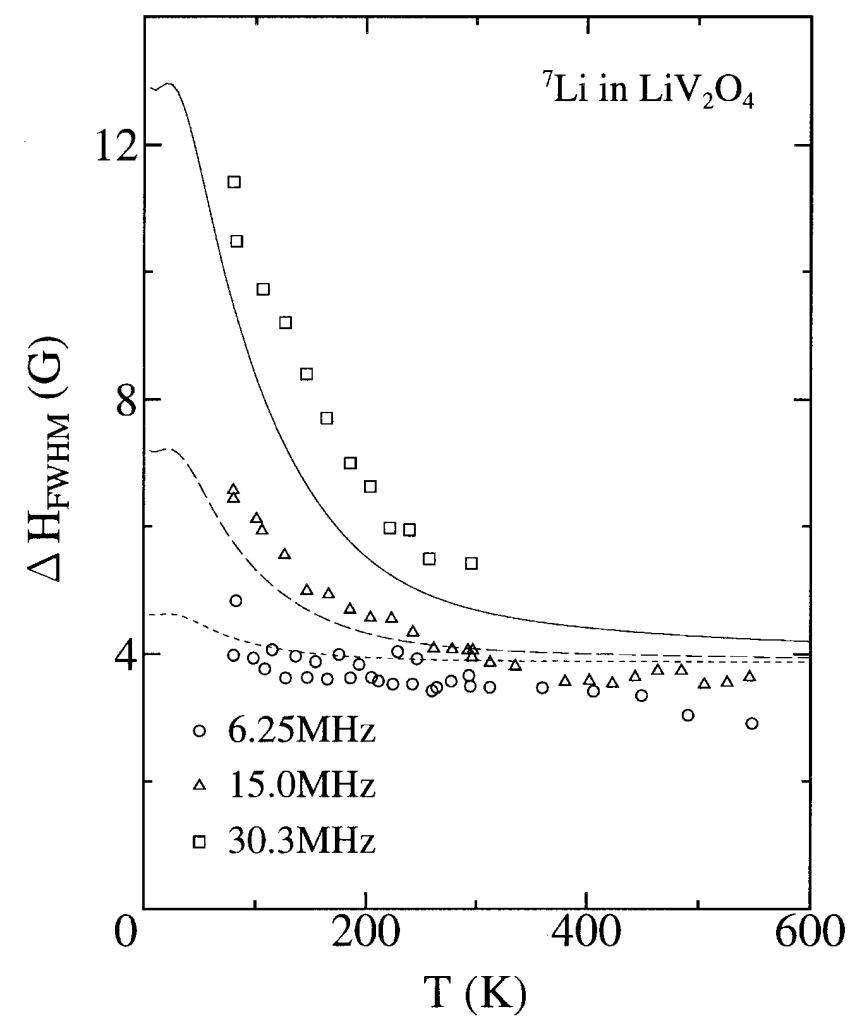

FIG. 11. Temperature dependence of the ${ }^{7} \mathrm{Li} \mathrm{NMR}$ full width at the half maximum $\Delta H_{\mathrm{FWHM}}$ in $\mathrm{LiV}_{2} \mathrm{O}_{4}$ at $6.25,15.0$, and $30.3 \mathrm{MHz}$, where the dotted, dashed, and solid lines indicate the calculated results with parameters provided in the Appendix.

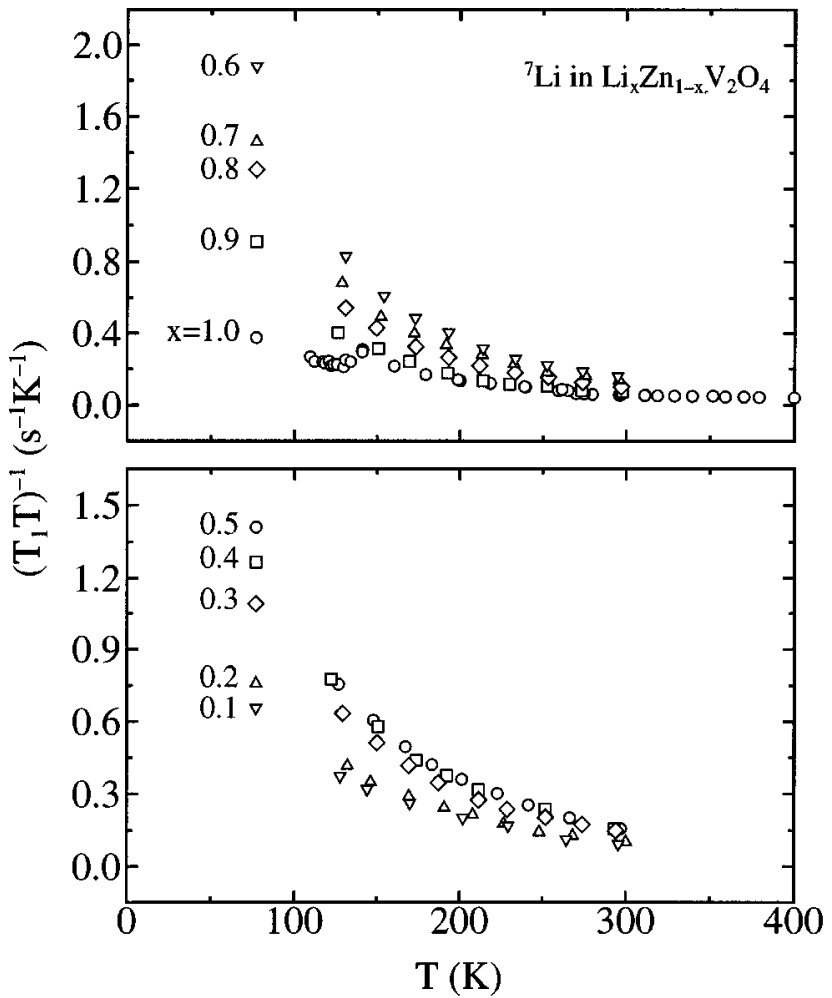

FIG. 12. Temperature dependence of the $\left(T_{1} T\right)^{-1}$ of ${ }^{7} \mathrm{Li}$ nuclei in $\mathrm{Li}_{x} \mathrm{Zn}_{1-x} \mathrm{~V}_{2} \mathrm{O}_{4}$ at $19.8 \mathrm{MHz}$, where $T_{1}$ is the spin-lattice relaxation time.

Here, the Lorentz-polarization correction has been applied to the intensities and the scattering factors of neutral atoms inferred from Ref. 25. All Debye-Waller factors are taken as zero and an occupancy probability corresponding to the chemical formula is assumed. The top of Fig. 13 indicates the $x$ dependence of $u$. The dotted, solid, and dashed lines in Fig. 2 are the calculated results for $u=0.3855,0.3865$, and 0.3875 , respectively. Thus, $u$ of the LMVO is found in the range of $u=0.3865(10)$. It is comparable to those of other normal vanadium spinels and the superconducting titanium compound; for $\mathrm{MgV}_{2} \mathrm{O}_{4}, u=0.3848(4),{ }^{26}$ for a $\mathrm{ZnV}_{2} \mathrm{O}_{4}$ single crystal, $u=0.3855(1),{ }^{27}$ and for a $\mathrm{LiTi}_{2} \mathrm{O}_{4}$ single crystal, $u=0.3872(4){ }^{28}$ Thus, it is concluded that neither the crystal symmetry nor the oxygen configuration varies in relation to $x$ and the change in the lattice constant on $x$ is expected to be directly attributed to that in the V-O bond length.

Since the V-V distances are larger than $2.91 \AA$, we may neglect the direct overlap of the vanadium $3 d$ wave functions for simplicity. In this case, the electronic state of LMVO or LZVO may be discussed based on the critical overlap integral between the vanadium and oxygen atoms. Using the above lattice constants and $u$ parameters, the average valence $v$ of the $\mathrm{V}$ ion is estimated to be as shown at the bottom of Fig. 13 in terms of the bond length $D(s)$ versus bond strength $s$ relation, ${ }^{29} D(s)=1.790-0.319 \mathrm{ln} s$, with $D(s)=(5 / 8-u) a$. In this figure, $v$ expected from the chemical formula, that is, $v=3+x / 2$, is also plotted by the dotted line. The real V-O bond length explains well an average valence of the $\mathrm{V}$ ion. 

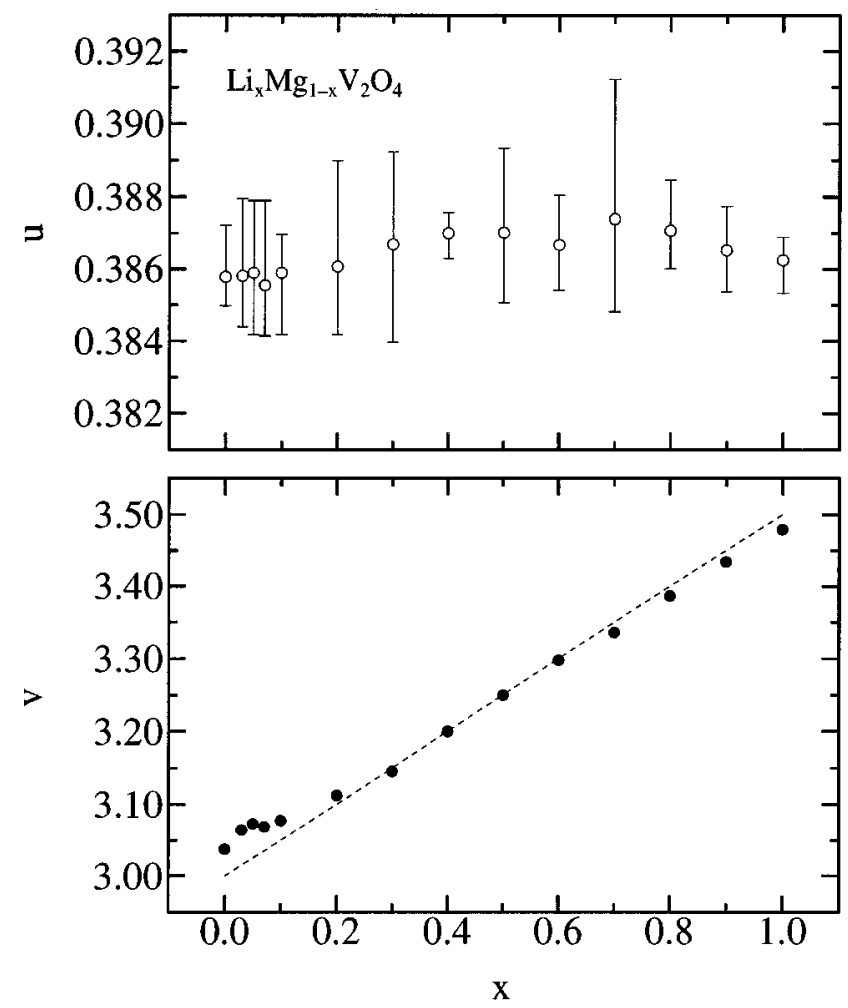

FIG. 13. $x$ dependence of the $u$ parameter and the vanadium valence $v$ of $\mathrm{Li}_{x} \mathrm{Mg}_{1-x} \mathrm{~V}_{2} \mathrm{O}_{4}$, where the dotted line indicates the valence expected from the chemical formula.

At first sight, the above conclusion seems to be inconsistent with previous NMR results for the LZVO around $x=0.4 .{ }^{13}$ Here, two kinds of electronic pathways, metallic and insulating domains, have been suggested to coexist. The correlation time of carriers that hop between these pathways is likely slower than the inverse of the NMR frequency, or the coherence length of the insulating domain is not so long. In order to clarify these points, $\mathrm{x}$-ray or neutron diffuse scattering experiments for single-crystal specimens are necessary. In the LMVO, we have observed only one NMR line of the $\mathrm{V}$ ion for the entire $x$ region whose linewidth depends largely on $x .^{22}$

\section{B. Transport properties}

In Sec. III B, the MIT point of LMVO was found to be $x_{c}=0.4$. For $x<0.4$, the transport mechanism of hole carriers is the VRH type. Here, the site randomness for the $\mathrm{Li}_{x} \mathrm{Mg}_{1-x}$ ion is considered to cause major effects. The longrange Coulomb interaction between carriers seems to be negligible from consideration of the temperature dependence of electrical resistivity. ${ }^{30}$

Let us estimate a critical exponent $\gamma$ for the coherence length $\xi$ in the wave function of carriers. ${ }^{31}$ The thermoelectric power on the VRH is expected to be proportional to $\xi^{-3 / 2}{ }^{16}$ Then, $\xi^{-1}$ as a function of $x$ is plotted at the bottom of Fig. 4(b) using the data at $290 \mathrm{~K}$. From the relation $\xi^{-1}$ $\propto\left|x-x_{c}\right|^{\gamma}$, where $x_{c}=0.4$ and $0<x<0.4$, we obtain $\gamma$ $=1.3$ with the solid line. This $\gamma$ value corresponds with the theory. ${ }^{31}$ The resistivity analysis has also indicated the decrease of $\xi^{-1}$ with $x$, but its $x$ dependence is not discussed simply because of the loosely bound specimens. Thus, it is confirmed that the MI transition in $\mathrm{Li}_{x} \mathrm{Mg}_{1-x} \mathrm{~V}_{2} \mathrm{O}_{4}$ is Anderson type.

For $x \geqslant 0.4$, the metallic conduction area may increase with $x$. Here, the fact that the electrical resistivity for $x$ $=0.6$ and 0.7 appears to be fitted by the VRH model above $160 \mathrm{~K}$ would imply that the random potential effect is still active. In $0.4 \leqslant x \leqslant 0.6$, the thermoelectric power is gradually transferred from its positive sign to negative with decreasing temperature. The $S$ data for $x=0.7$ are in the range between -2 and $2 \mu \mathrm{V} / \mathrm{K}$. In $0.8 \leqslant x \leqslant 1, S$ is negative and positive above and below $300 \mathrm{~K}$, respectively. Thus, this suggests two kinds of carriers in the metallic phase. This could be attributed to $(2-x) \mathrm{V}^{3+}$ and $x \mathrm{~V}^{4+}$ ions with a dynamic fluctuation, which behave like electrons and holes, respectively. Therefore, $S$ is expressed as $S=\left(\sigma_{e} S_{e}+\sigma_{h} S_{h}\right) /\left(\sigma_{e}+\sigma_{h}\right)$, where $\sigma$ is the conductivity and the subscripts $e$ and $h$ refer to the electron and hole, respectively. This idea should be tested by another experiment. The peak at about $60 \mathrm{~K}$ in $\mathrm{LiV}_{2} \mathrm{O}_{4}$ with no random potential denotes the transport anomaly of hole carriers of $\mathrm{V}^{4+}$ ions.

\section{Magnetic properties}

As shown in Fig. $7, \chi_{d}^{-1}$ in the region below $500 \mathrm{~K}$ deviates from the Curie-Weiss law with parameters obtained from the analysis at high temperatures.

The previous work based on a high-temperature-series expansion analysis has already shown that the deviation at low temperatures in insulating $\mathrm{MgV}_{2} \mathrm{O}_{4}$ is due to the exchange field between spins larger than temperature or short-range ordered spin correlation. ${ }^{8,9}$ Here, the Curie constant should be given by the Hund's rule in all temperature regions. Such an effect has been confirmed in the semiconducting perovskite $\mathrm{LaVO}_{3}$ as well. ${ }^{32}$ It has also been pointed out that in $\mathrm{Mg}\left(\mathrm{V}_{1-x} \mathrm{Al}_{x}\right)_{2} \mathrm{O}_{4}$ with $0.1<x \leqslant 0.25$, short-range ordered clusters with a superparamagnetic effect exist. ${ }^{8,9}$

Keeping the above factors in mind, the susceptibility results below $500 \mathrm{~K}$ can be classified as (i) $0 \leqslant x \leqslant 0.05$, (ii) $0.07 \leqslant x \leqslant 0.3$, and (iii) $0.4 \leqslant x \leqslant 1$. The susceptibilities in cases (i) and (ii) have tendencies similar to those of $\mathrm{MgV}_{2} \mathrm{O}_{4}$ and $\operatorname{Mg}\left(\mathrm{V}_{1-x} \mathrm{Al}_{x}\right)_{2} \mathrm{O}_{4}$, respectively. On the other hand, the compounds with $0.4 \leqslant x \leqslant 1$ are essentially metals.

In $0 \leqslant x \leqslant 0.05$ [case (i)], the antiferromagnetic transition appears, where $T_{N}$ may correspond nearly to that at which the susceptibility has a sharp edge. Actually, the NMR line of $\mathrm{V}$ ions in $\mathrm{MgV}_{2} \mathrm{O}_{4}$ disappears below $T_{N}$ and a large specific-heat anomaly appears. ${ }^{8,9}$ Moreover, from x-ray powder diffraction of $\mathrm{MgV}_{2} \mathrm{O}_{4}$ at low temperatures, it has been found that at $T_{N}$ the cubic-tetragonal structural transition occurs. ${ }^{9}$ Therefore, the transition for $0 \leqslant x \leqslant 0.05$ may be attributed to the removal of degenerated antiferromagnetic spin configuration. $T_{N}$ decreases rapidly with increasing $x$ as shown by open circles in Fig. 14. On one hand, the reduction of $T_{N}$ may be expected from the fact that the Weiss temperature at high temperatures decreases with increasing $x$, although its $x$ dependence is considerably larger than that expected from the Weiss temperature. Another origin is that the coherence length of the antiferromagnetic state may be reduced by carrier doping, because the present results of thermoelectric power in $0<x<0.4$ have indicated that the coher- 


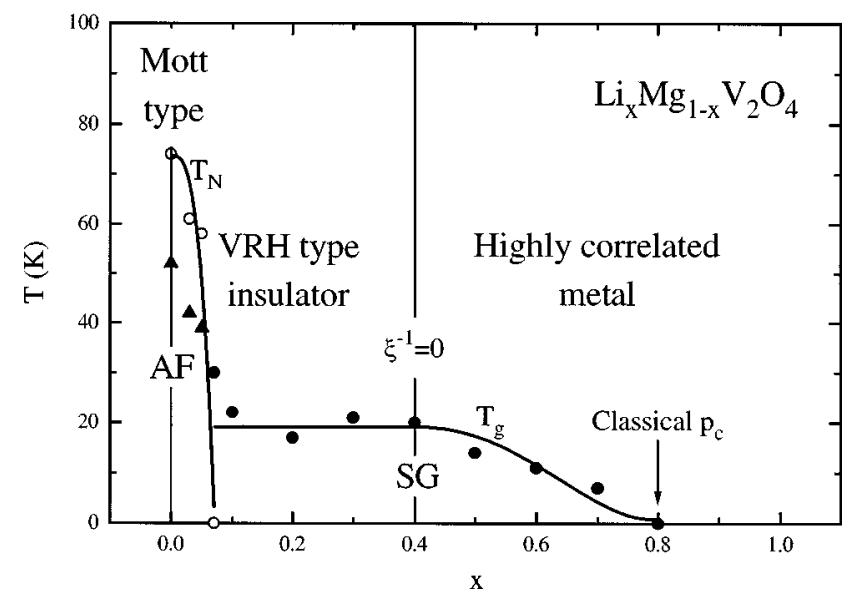

FIG. 14. Phase diagram of $\mathrm{Li}_{x} \mathrm{Mg}_{1-x} \mathrm{~V}_{2} \mathrm{O}_{4}$. AF is the antiferromagnetic phase below $T_{N}$ (open circles) and SG is the spin-glass phase below $T_{g}$ (solid circles). The solid triangles indicate apparent $T_{g}$ for the compounds whose susceptibility takes the sharp edge as well as the broad peak. The classical percolation threshold $p_{c}$ and the concentration at which the coherence length $\xi$ in wave function of carriers diverges are also indicated. The solid lines are drawn for guides to the eye.

ence length in the wave function of carriers increases with $x$. In $0<x \leqslant 0.05$, it is natural to think that the carrier-rich and carrier-poor clusters originate from the $\mathrm{V}$ sites located near $\mathrm{Li}^{+}$and $\mathrm{Mg}^{2+}$, respectively.

As denoted by solid triangles in Fig. $14, T_{g}$, at which the broad peak of susceptibility appears, with the remanent magnetization only in the FC condition decreases with $x$, which appears to correlate with $T_{N}$. The behavior at $T_{g}$ may remind us of the spin-glass phase. ${ }^{11}$ Here, it has been known that the ground states of three-dimensional frustrated spin systems are likely "cooperative paramagnet,",11 "quantum spin liquid," ${ }^{33}$ or " "spin-glass." ${ }^{34}$ However, in $\mathrm{MgV}_{2} \mathrm{O}_{4}$, the structural transition occurs at $T_{N}$ and the antiferromagnetic lattice exists at $4.2 \mathrm{~K}$. In addition, the magnitude of remanent magnetization at $4.2 \mathrm{~K}$ is considerably small and the broad peak is not suppressed even by a magnetic field of $80 \mathrm{kG}{ }^{9}$ Thus, we may have to consider complex spin states or successive transitions through an exchange distortion possibly due to the Jahn-Teller effect. Of course, it is also necessary to explore possible inhomogeneity of atoms microscopically.

In $0.07 \leqslant x \leqslant 0.3$ [case (ii)], $T_{N}$ disappears and only the broad peak of susceptibility appears at $T_{g}$. Below $T_{g}$, significant remanent magnetization has been found only in the FC condition. This suggests the appearance of a spin-glass phase. As shown in Fig. 7, the susceptibility has a Curie-like temperature dependence between $T_{g}$ and $50 \mathrm{~K}$. Here, the Curie constant in this temperature region is smaller than that at higher temperatures. This behavior may be attributed to the formation of short-range ordered clusters with the superparamagnetic effect. In effect, the spin correlation develops and the uncompensated clusters grow gradually as the temperature is lowered. The spin-glass phase is considered to appear after the freezing of the clusters. The fact that $T_{g}$ for the compounds with significant remanent magnetization does not depend on $x$ as shown by solid circles in Fig. 14 suggests that the effective coupling constant relevant to the spin-glass phase is originated from the superexchange.
In $0.4 \leqslant x \leqslant 1$ [case (iii)], $\chi_{d}^{-1}$ below $500 \mathrm{~K}$ is smaller than the extrapolated value from the Curie-Weiss law at high temperatures. Between 100 and $400 \mathrm{~K}, \chi_{d}^{-1}$ appears to have zero or a small ferromagnetic exchange constant. It strongly suggests that $500 \mathrm{~K}$ for the metallic compounds may correspond to a degeneracy-nondegeneracy transition temperature that is roughly estimated to be $T^{*} \simeq E_{F} / \alpha$, with $E_{F}$ and $\alpha$ being the Fermi energy and the mass enhancement factor, respectively. For the temperature dependence of $\chi_{d}$ below $400 \mathrm{~K}$, Stoner or a self-consistent renormalization (SCR) model $^{35}$ would be candidates. Although the behavior of $\chi_{d}^{-1}$ is similar to that expected from the model, they cannot explain the low degeneracy temperature. An alternative model may be the Brinkman-Rice type that leads to the reduction of $E_{F}$ or the enhancement of effective mass. ${ }^{36}$ However, it cannot introduce nearly zero exchange constant. Perhaps, the Stoner (or SCR) model for the electron system with enhanced mass is necessary.

In the region near $T_{g}$, for the compounds with $0.4 \leqslant x$ $\leqslant 0.6, \chi_{d}^{-1}$ decreases rapidly with decreasing temperature. This is related to the occurrence of the remanent magnetization under the FC condition, that is, the spin freezing. Above $x=0.8$, no such tendency is observed, which may be consistent with the fact that these compounds are metallic.

For simplicity, let us consider the carrier-rich and carrierpoor clusters in the atomic scale, to explain qualitatively the $x$ dependence of remanent magnetization in Fig. 6(b). The carrier-poor cluster cannot form the long-range ordered antiferromagnetic state above $x=0.07$, but it may have localized and paramagnetic spins near the boundary between the clusters. The freezing of these clusters contributes to the remanent magnetization under the FC condition. As the carrier concentration increases, both the surface of the carrier-poor clusters and the localized spins will increase. With increasing the carrier concentration further, the expansion of these clusters appears to take a maximum at a certain concentration, probably less than $x_{c}=0.4$. When the carrier-rich clusters overlap significantly, or the crystals are covered with the carriers completely to form metals, the carrier-poor cluster cannot be formed and thus the remanent magnetization disappears.

\section{Microscopic properties}

The result that only one $\mathrm{V}$ NMR spectrum of $\mathrm{LiV}_{2} \mathrm{O}_{4}$ exists down to $10 \mathrm{~K}$ with no significant line broadening indicates that a dynamic mixed valence state of $\mathrm{V}^{3+}$ and $\mathrm{V}^{4+}$ ions exists, as indicated by the structure analysis, a longrange ordered state is absent, and the crystal symmetry remains cubic.

The Knight shift of ${ }^{51} \mathrm{~V}$ nuclei is expressed as $K=K_{d}$ $+K_{\text {orb }}$, where the first and second terms are contributions from the $d$ spin and the orbital paramagnetism, respectively. These terms are related to the respective susceptibilities as $K_{i}=H_{\mathrm{hf}}^{i} \chi_{i} /\left(N \mu_{B}\right)$ through the hyperfine field $H_{\mathrm{hf}}^{i}$. The solid line in the $K-\chi$ diagram shown in the inset of Fig. 8 provides $H_{\mathrm{hf}}^{d}=-74 \mathrm{kG} / \mu_{B}$. Taking the $\chi_{\text {orb }}$ value obtained from the analysis of the high-temperature susceptibility in Sec. III C, we get $H_{\mathrm{hf}}^{\text {orb }}=350 \mathrm{kG} / \mu_{B}$ and $K_{\text {orb }}=0.55 \%$. The following $H_{\mathrm{hf}}^{d}$ values have been found for conducting $\mathrm{V}$ oxides and bronzes; for $\mathrm{V}^{4+}$ ions, $H_{\mathrm{hf}}^{d}=-85 \mathrm{kG} / \mu_{B}$ in 
$\mathrm{VO}_{2}$ (Ref. 37) and $\beta-\mathrm{Na}_{x} \mathrm{~V}_{2} \mathrm{O}_{5}$ (Ref. 38) and for $\mathrm{V}^{3+}$ ions, $H_{\mathrm{hf}}^{d}=-120 \mathrm{kG} / \mu_{B}$ in $\mathrm{V}_{2} \mathrm{O}_{3}$ (Ref. 37) or $-60 \mathrm{kG} / \mu_{B}$ in $\mathrm{LiVO}_{2} \cdot{ }^{39}$ Thus, the covalent admixture with the oxygen $2 p$ orbital for $\mathrm{V}$ ions in $\mathrm{LiV}_{2} \mathrm{O}_{4}$ is expected to be larger than those in $\mathrm{VO}_{2}$ or $\mathrm{V}_{2} \mathrm{O}_{3}$. Similar behavior has been seen in the intermetallic compound $\mathrm{V}_{3} \mathrm{Si}$ having a high conductivity. In this case, $H_{\mathrm{hf}}^{d}$ of the $\mathrm{V}$ nuclei is reduced to about $-25 \mathrm{kG} / \mu_{B} .^{40}$

The Li Knight shift of $\mathrm{LiV}_{2} \mathrm{O}_{4}$ is found to be nearly proportional to the susceptibility in the measured temperature region as found in the inset of Fig. 10. The field of $\mathrm{Li}$ ions is considered to be caused by a transferred field $H_{\mathrm{hf}}^{\mathrm{tr}}$ from the $3 d$ electron of $\mathrm{V}$ ions. Thus, the Knight shift is written as $K=K_{0}+H_{\mathrm{hf}}^{\mathrm{tr}} \chi /\left(N \mu_{B}\right)$, where $K_{0}$ is the susceptibilityindependent Knight shift. From the $K-\chi$ diagram, $K_{0}$ and $H_{\mathrm{hf}}^{\mathrm{tr}}$ are estimated to be $1 \times 10^{-3} \%$ and $1.6 \mathrm{kG} / \mu_{B}$, respectively. This $K_{0}$ is an order of magnitude smaller than that of $\mathrm{Li}$ metal, ${ }^{41}$ which indicates that the $\mathrm{Li}$ atoms in $\mathrm{LiV}_{2} \mathrm{O}_{4}$ are completely ionized and the outer $2 s$ electrons are transferred to the $\mathrm{V}$ orbitals. The origin of the NMR linewidth for $\mathrm{Li}$ ions is discussed in the Appendix.

The spin-lattice relaxation rate of ${ }^{51} \mathrm{~V}$ nuclei in $\mathrm{LiV}_{2} \mathrm{O}_{4}$ is considered to be mainly due to the fluctuation of hyperfine coupling field. Based upon a fluctuationdissipation theorem, $\left(T_{1} T\right)^{-1}$ is written as $\left(T_{1} T\right)^{-1}$ $=\gamma_{\mathrm{v}}^{2} N^{-2} \Sigma\left|A_{q}\right|^{2} \operatorname{Im}\left\{\chi^{-+}\left(q, \omega_{0}\right)\right\} / \omega_{0}$, where $\gamma_{\mathrm{v}}$ is the gyromagnetic ratio of the ${ }^{51} \mathrm{~V}$ nuclei, $A_{q}$ is the hyperfine coupling constant with respect to the wave number $q$, $\operatorname{Im}\left\{\chi^{-+}\left(q, \omega_{0}\right)\right\}$ is the perpendicular component to the direction of local field in the imaginary part for the dynamical susceptibility, and $\omega_{0}$ is the resonance frequency. ${ }^{42}$ In the measured temperature region, $\left(T_{1} T\right)^{-1}$ appears to have the Curie-Weiss-type temperature dependence, $\left(T_{1} T\right)^{-1} \propto(T$ $-26)^{-1}$, as shown by the dotted line in Fig. 9. It reminds us of a model for nearly ferromagnetic metals based on SCR theory. ${ }^{35}$ Between 100 and $400 \mathrm{~K}, \chi_{d}$ appears to have a weak ferromagnetic spin correlation, but obviously, $\left(T_{1} T\right)^{-1}$ versus the static susceptibility plot shown in the inset of Fig. 9 does not provide a reasonable intercept. It is necessary to construct a modified SCR theory, at least from the viewpoint described in the previous section.

On the other hand, at high temperatures where the CurieWeiss law and Hund's rule hold, the magnetic excitation is expected to be similar to that of insulators. In this case, $T_{1}{ }^{-1}$ is temperature independent and approximately written as $T_{1}^{-1}=\sqrt{2 \pi} g^{2} \gamma_{v}^{2} H_{\mathrm{hf}}^{d 2} S(S+1) /\left(3 \omega_{e}\right)$, where $\omega_{e}$ is the exchange frequency expressed as $\omega_{e}^{2}=8 J^{2} z S(S+1) /\left(3 \hbar^{2}\right), \hbar$ and $z$ being Planck's constant and the number of nearest V-V pairs, respectively. ${ }^{43}$ Taking the values at high temperatures for $g, J$, and $S(S+1)$, we get $T_{1}^{-1}=3.7 \times 10^{4} \mathrm{~s}^{-1}$, which is close to the extrapolated value.

Since $T_{1}$ of ${ }^{7} \mathrm{Li}$ nuclei is independent of the Larmor frequency, Li ionic motion is probably absent in the measured temperature region, which is consistent with an observation of an almost Gaussian line shape. It has already been shown that the Knight shift of the Li ion of the LZVO and LMVO is caused by the transferred field from the $3 d$ electrons of the $\mathrm{V}$ ion. ${ }^{13,22}$ Neglecting the $q$ dependence on $A_{q},\left(T_{1} T\right)^{-1}$ may be written as $\left(T_{1} T\right)^{-1}=\left(H_{\mathrm{hf}}^{\mathrm{tr}} / H_{\mathrm{hf}}^{d}\right)^{2}\left(T_{1} T\right)_{\mathrm{v}}^{-1}$, where $\left(T_{1} T\right)_{\mathrm{v}}^{-1}$ is for the $\mathrm{V}$ ion. Actually, in the case of $\mathrm{LiV}_{2} \mathrm{O}_{4}$, using the above parameters, $\left(T_{1} T\right)^{-1}$ of $\mathrm{Li}$ ion is found to be comparable to that of the $\mathrm{V}$ ion. However, this idea cannot explain the anomalies at 140 and $270 \mathrm{~K}$, because no corresponding anomalies have been seen for $\left(T_{1} T\right)_{\mathrm{v}}^{-1}$. It could be related to a slight deficiency of $\mathrm{Li}$ atoms. For other compounds in the LZVO, $\left(T_{1} T\right)^{-1}$ has roughly the Curie-Weisstype temperature dependence as in $\left(T_{1} T\right)_{\mathrm{v}}^{-1}$ of $\mathrm{LiV}_{2} \mathrm{O}_{4}$. Around $x=0.4$ of the LZVO, two kinds of V NMR lines and only one Li line have been observed. ${ }^{13}$ Therefore, the NMR relaxation of the $\mathrm{Li}$ ion may be due to the fluctuation of an average local field from the metallic and insulating clusters. The fact that $\left(T_{1} T\right)^{-1}$ has a similar temperature dependence irrespective of whether the compound is metallic or insulating suggests that the magnetic excitations for two clusters are similar and/or the $\mathrm{V}$ ions near $\mathrm{Li}$ form the metallic pathway in the VRH regime.

\section{CONCLUSION}

We have explored spin fluctuation and transport mechanism in $\mathrm{Li}_{x} \mathrm{Mg}_{1-x} \mathrm{~V}_{2} \mathrm{O}_{4}$ and $\mathrm{Li}_{x} \mathrm{Zn}_{1-x} \mathrm{~V}_{2} \mathrm{O}_{4}$ through measurements of $\mathrm{x}$-ray diffraction, electrical resistivity, thermoelectric power, magnetization, and NMR.

In Sec. III, we have summarized the results referring to previous analysis procedures. In Sec. IV, new items revealed in this work have been described and the phase diagram of the LMVO at low temperatures presented.

Within an experimental error, the trigonal distortion for the $\mathrm{VO}_{6}$ octahedra is unchanged for the entire $x$ region at room temperature. Thus, one may consider a mechanism of the metal-insulator transition as a function of hole concentration and random potential. Only $\mathrm{MgV}_{2} \mathrm{O}_{4}$ is a broadband semiconductor and Mott-type insulator. An introduction of hole carriers leads to the VRH-type phase in the region 0 $<x<0.4$. The MIT point of LMVO is $x_{c}=0.4$, which is about one-half of the classical site percolation threshold $p_{c}$. The $x$ dependence of coherence length $\xi$ estimated from the thermoelectric power for the insulating compounds may be consistent with the MIT in this system being an Anderson transition. The metallic phase likely has two kinds of carriers from a dynamic mixed valence state of $\mathrm{V}^{3+}$ and $\mathrm{V}^{4+}$, which behave as electrons and holes, respectively.

In the region above $500 \mathrm{~K}$, the magnetic properties of LMVO have been explained by a magnetically localized electron model with $J=54 \mathrm{~K}$ irrespective of whether the system is metallic or insulating. This strongly suggests that the characteristic temperature of $500 \mathrm{~K}$ corresponds to the degeneracy-nondegeneracy transition temperature for the metallic compounds. The Fermi energy of the metallic compounds is largely reduced possibly due to the large enhancement of effective mass. Thus, the metallic properties of LMVO and LZVO have been discussed from the viewpoint of a highly correlated electron system, where spin fluctuations determine the total density of states. In order to understand the temperature dependence of spin susceptibility and spin-lattice relaxation time for the metallic compounds, we may have to construct the modified Stoner (or SCR) model for the correlated electron system with large mass enhancement. From the viewpoint of a localized electron model, on the other hand, an interaction similar to a double exchange effect $^{44,45}$ would be a candidate, where the ferromagnetic exchange coupling is expected to be effectively induced in the 
antiferromagnetically correlated lattice. Two kinds of carriers in the metallic phase as suggested above originate from highly correlated electronic states. The anomalies of thermoelectric power and spin susceptibility at low temperatures in $\mathrm{LiV}_{2} \mathrm{O}_{4}$ with no site randomness may be caused by the pseudogaplike state for the carrier density and by spin fluctuations, respectively.

Analysis of our data for LMVO leads to the phase diagram at low temperatures of Fig. 14 with several critical parameters and properties described above. Other parameters are summarized as follows: (a) In the Mott insulator and the VRH type insulator compounds, short-range ordered spin correlation and/or superparamagnetic effects appear. $T_{N}$ is the Néel temperature below which long-range antiferromagnetic (AF) order occurs for $x \leqslant 0.05$ accompanied with the cubic-tetragonal structural transition originally driven by the Jahn-Teller effect. This is based on the results of magnetic susceptibility, low-temperature $\mathrm{x}$-ray diffraction, ${ }^{9}$ specific heat, ${ }^{9}$ and NMR. ${ }^{8}$ (b) $T_{g}$ is the temperature below which the spin-glass SG phase appears in the region of $0.07 \leqslant x \leqslant 0.7$ with a significant remanent magnetization only in the FC condition due to the frustration inherent in the spinel $B$ lattice. This phase is enhanced in the vicinity of $x=0.3$, because a surface of the carrier-poor cluster may reach a maximum in the concentration slightly less than $x_{c}$.

We have pointed out the magnetic anomalies with very small remanent magnetizations for $0 \leqslant x \leqslant 0.05$, whose temperature is shown by solid triangles in Fig. 14. Analysis of magnetic properties at high temperatures has also indicated the difference between Van Vleck orbital susceptibilities above and below $x_{c}$. Precise experiments on the electronic structures are desired.

\section{ACKNOWLEDGMENTS}

We thank Professor N. Tsuda for supplying us with the specimens of $\mathrm{Li}_{x} \mathrm{Zn}_{1-x} \mathrm{~V}_{2} \mathrm{O}_{4}$ and Professor D. S. Hirashima for valuable discussions.

\section{APPENDIX}

We shall consider the origin of the NMR linewidth of ${ }^{7} \mathrm{Li}$ nuclei in $\mathrm{LiV}_{2} \mathrm{O}_{4}$. The NMR linewidth in paramagnetic materials is generally caused by several interactions between the investigated nucleus and its surroundings. The second moments from the internuclear interaction and from the magnetic field of the neighboring powder grains have been found to give appreciable effects for the linewidth. According to the Van Vleck formula, ${ }^{46}$ the contributions from the Li-Li and $\mathrm{Li}-\mathrm{V}$ interactions are 0.67 and $2.02 \mathrm{G}^{2}$, respectively, with $a=8.2341 \AA$. Another contribution from the magnetic field of the neighboring powder grains is estimated to be $2.30 \times 10^{-3}\left(\chi H_{0}\right)^{2}, H_{0}$ being the resonance field, from the model proposed by Drain, ${ }^{47}$ with the assumption of cubic close packing of spherical particles. The calculated results at 6.25, 15.0, and $30.3 \mathrm{MHz}$ are shown in Fig. 11 by the dotted, dashed, and solid lines, respectively. An agreement between the experimental and calculated results is satisfactory, except for the region above $500 \mathrm{~K}$. A deviation from the calculated curve in the high-temperature region is possibly due to the onset of $\mathrm{Li}$ ionic motion.
*Present address: Faculty of Science, Shinshu University, Matsumoto 390, Japan.

${ }^{1}$ N. F. Mott, Metal-Insulator Transitions, 2nd ed. (Taylor \& Francis, London, 1990).

${ }^{2}$ A. Georges, G. Kotliar, W. Krauth, and M. J. Rozenberg, Rev. Mod. Phys. 68, 13 (1996).

${ }^{3}$ For example, F. S. Galasso, Structure and Properties of Inorganic Solids (Pergamon Press, Oxford, 1970), Chap. 8.

${ }^{4}$ For example, S. Krupička and P. Novák, Ferromagnetic Materials, Vol. 3 (North-Holland, Amsterdam, 1982), Chap. 4.

${ }^{5}$ D B. Rogers, J. L. Gillson, and T. E. Gier, Solid State Commun. 5, 263 (1967).

${ }^{6}$ A. Fujimori, K. Kawakami, and N. Tsuda, Phys. Rev. B 38, 7889 (1988)

${ }^{7}$ K. Kawakami, Y. Sakai, and N. Tsuda, J. Phys. Soc. Jpn. 55, 3174 (1986).

${ }^{8}$ H. Mamiya and M. Onoda, Solid State Commun. 95, 217 (1995).

${ }^{9}$ H. Mamiya, M. Onoda, T. Furubayashi, J. Tang, and I. Nakatani, J. Appl. Phys. 81, 5289 (1997).

${ }^{10}$ Yu. A. Izyumov, V. E. Naish, and S. B. Petrov, J. Magn. Magn. Mater. 13, 267 (1979).

${ }^{11}$ J. Villain, Z. Phys. B 33, 31 (1979).

12 Muhtar, F. Takagi, K. Kawakami, and N. Tsuda, J. Phys. Soc. Jpn. 57, 3119 (1988).

${ }^{13}$ Y. Amako, T. Naka, M. Onoda, H. Nagasawa, and T. Erata, J. Phys. Soc. Jpn. 59, 2241 (1990).

${ }^{14}$ R. D. Shannon, Acta Crystallogr. Sec. A 32, 751 (1976).

${ }^{15}$ N. F. Mott, Philos. Mag. 19, 835 (1969).

${ }^{16}$ W. Brenig, G. H. Döhler, and P. Wölfle, Z. Phys. 258, 381 (1973)
${ }^{17}$ For example, A. H. Wilson, The Theory of Metals, 2nd ed. (Cambridge University Press, London, 1958), Chap. 8.

${ }^{18}$ J. Jaskowsky, Ber. Bunsenges. Phys. Chem. 70, 199 (1966).

${ }^{19}$ J. B. Goodenough, Mater. Res. Bull. 5, 621 (1970).

${ }^{20}$ D. Fiorani, L. Gastaldi, A. Lapiccirella, and S. Viticoli, Solid State Commun. 32, 831 (1979).

${ }^{21}$ For example, E. König, and G. König, in Magnetic Properties of Coordination and Organometallic Transition Metal Compounds, edited by K.-H. Hellwege and A. M. Hellwege, LandoltBörnstein, New Series, Group II, Vol. 12 (Springer-Verlag, Berlin, 1984), Chap. 1.

${ }^{22} \mathrm{M}$. Onoda and K. Shigekawa (unpublished).

${ }^{23}$ H. Kessler and M. J. Sienko, J. Chem. Phys. 55, 5414 (1971)

${ }^{24}$ B. L. Chamberland and T. A. Hewston, Solid State Commun. 58, 693 (1986).

${ }^{25}$ International Tables for Crystallography Vol. IV, edited by J. A. Ibers and W. C. Hamilton (Kynoch Press, Birmingham, England, 1974).

${ }^{26}$ R. Plumier and A. Tardieu, C. R. Acad. Sci. 257, 3858 (1963).

${ }^{27} \mathrm{M}$. Onoda, unpublished results based on x-ray four-circle analysis.

${ }^{28}$ J. Akimoto, Y. Gotoh, K. Kawaguchi, and Y. Oosawa, J. Solid State Chem. 96, 446 (1992).

${ }^{29}$ W. H. Zachariasen, J. Less-Common Met. 62, 1 (1978).

${ }^{30}$ A. L. Efros and B. I. Shklovskii, J. Phys. C 8, L49 (1975).

${ }^{31}$ For a review, B. Kramer and A. MacKinnon, Rep. Prog. Phys. 56, 1469 (1993).

${ }^{32}$ M. Onoda and H. Nagasawa, Solid State Commun. 99, 487 (1996). 
${ }^{33}$ M. Mekata, T. Asano, T. Sugino, H. Nakamura, N. Asai, M. Shiga, A. Keren, K. Kojima, G. M. Luke, W. D. Wu, Y. J. Uemura, S. Dunsinger, and M. Gingras, J. Magn. Magn. Mater. 140-144, 1767 (1995).

${ }^{34}$ M. J. P. Gingras, C. V. Stager, B. D. Gaulin, N. P. Raju, and J. E. Greedan, J. Appl. Phys. 79, 6170 (1996).

${ }^{35}$ T. Moriya, Spin Fluctuations in Itinerant Electron Magnetism (Springer-Verlag, Berlin, 1985).

${ }^{36}$ W. F. Brinkman and T. M. Rice, Phys. Rev. B 2, 4302 (1970).

${ }^{37}$ For example, A. C. Gossard, J. P. Remeika, T. M. Rice, H. Yasuoka, K. Kosuge, and S. Kachi, Phys. Rev. B 9, 1230 (1974).

${ }^{38}$ M. Onoda and H. Nagasawa, Phys. Status Solidi B 141, 507
(1987), and references therein.

${ }^{39}$ M. Onoda and T. Inabe, J. Phys. Soc. Jpn. 62, 2216 (1993), and references therein.

${ }^{40}$ A. M. Clogston, A. C. Gossard, V. Jaccarino, and Y. Yafet, Phys. Rev. Lett. 9, 262 (1962).

${ }^{41}$ W. D. Knight, Solid State Phys. 2, 122 (1956).

${ }^{42}$ T. Moriya, J. Phys. Soc. Jpn. 43, 516 (1963).

${ }^{43}$ T. Moriya, Prog. Theor. Phys. 16, 641 (1956).

${ }^{44}$ P.-G. de Gennes, Phys. Rev. 118, 141 (1960).

${ }^{45}$ P. W. Anderson and H. Hasegawa, Phys. Rev. 100, 675 (1955).

${ }^{46}$ For example, A. Abragam, The Principles of Nuclear Magnetism (Oxford University Press, Oxford, 1961), Chap. 4.

${ }^{47}$ L. E. Drain, Proc. Phys. Soc. London 80, 1380 (1962). 